1. PhD in Economics, University of York, Associate Professor in Economics, Dipartimento di Economia, Università di Trento and IDEC.

2. Full Professor in Applied Economics, Dipartimento di Economia, Università di Trento and IDEC.

3. Research Fellow, Fondazione per la Sussidiarietà. 


\title{
POLITICHE E AIUTI ALLO SVILUPPO: CHE COSA ABBIAMO IMPARATO?
}

\author{
POLITICS AND AID TO DEVELOPMENT: \\ WHAT WE HAVE LEARNED?
}

\author{
Gabriella Berloffa ${ }^{1}$ \\ Giuseppe Folloni² \\ Ilaria Schnyder von Wartensee ${ }^{3}$
}

\begin{abstract}
This paper analyzes and discusses the empirical evidence of low effectiveness in the growth of policies of investment in physical and human capital based on international aid ( $\$ 1$ and 2). Reasons for that are linked both to the limits of analytical and econometric methods (§ 4) and to the existence of strong complementarities between different dimensions of the macroeconomic, social and institutional context ( $\S 3$ ). Proposed new strategies to gain effectiveness in the development of projects and policies are critically discussed.
\end{abstract}

Key words: Aid effectiveness; Growth policies; Institutions; Development. 


\section{Introduzione}

Per molto tempo è stato naturale pensare che la crescita economica fosse il modo con cui si manifestava lo sviluppo di una società: il verificarsi di una sensibile e robusta crescita in un'economia significava che in quella società erano in atto dei cambiamenti culturali e di comportamento che permettevano la permanenza della crescita stessa. Tuttavia, spesso si è ritenuto che la "vicinanza" fra i due termini implicasse qualcosa di più. Rovesciando il nesso di causalità si è ritenuto per molto tempo che bastasse avviare processi di crescita quantitativa per far sì che le soggiacenti dimensioni di sviluppo fossero a loro volta implicate e messe in moto. Per molti anni perciò sia il mondo accademico che i governi hanno concentrato l'attenzione sulle varie "ricette" in grado di mettere in moto la crescita. L'obiettivo era trovare le modalità per garantire una crescita economica robusta $e$, di conseguenza, un cambiamento più generale delle condizioni di vita e quindi del benessere.

Analizzando i dati sulla crescita di lungo periodo (1870-2000), riportati da Maddison (2001), si nota che certi paesi sono cresciuti durante tutto il secolo scorso diventando sempre più ricchi (gruppo OECD), altri hanno cominciato a crescere a tassi sostenuti soprattutto nell'ultimo trentennio (il gruppo che Maddison chiama Resurgent Asia); altri ancora sono rimasti ai bassi livelli iniziali o addirittura le loro condizioni si sono deteriorate (I'Africa in particolare). Come mai alcuni gruppi di paesi hanno conosciuto fenomeni di crescita prolungata e stanno mostrando forme di convergenza verso livelli di PIL pro capite dei paesi sviluppati e altri no? A che cosa sono dovute queste disparità?

L'evidenza di diversi sentieri di crescita nelle differenti regioni ha acceso un forte dibattito sulle determinanti della crescita e sull'efficacia degli aiuti concessi ai paesi meno sviluppati. La mancata crescita di alcuni paesi veniva imputata ad una incapacità di investimento dovuta ai bassi risparmi (teorie del "gap finanziario"). L'azione delle Istituzioni Finanziarie Internazionali (IFI) è stata influenzata per lungo tempo dall'idea che il problema fondamentale dello sviluppo economico fosse eliminare il gap finanziario, cioè la mancanza di risorse per gli investimenti. "Few economic ideas are as intuitive as the notion that increasing investment is the best way to raise future output, either for an individual or a nation" (KING; LEVINE, 1994, p. 1). Occorreva perciò colmare questo "gap finanziario" attraverso aiuti internazionali.

L'approccio del gap financing, per quanto attraente nella sua semplicità intuitiva, ha mostrato tuttavia nel tempo la sua inadeguatezza sotto il profilo sia teorico sia empirico. Dal punto di vista teorico è emersa l'importanza di altri fattori quali il capitale umano e si è posta sempre più attenzione alla qualità del contesto macroeconomico e socio-istituzionale dei paesi quale condizione necessaria perché vengano avviati processi di crescita consistenti e quindi per l'efficacia degli aiuti stessi. Dal punto di vista empirico, molti studi hanno cercato di verificare direttamente la relazione tra le due variabili, aiuti e crescita, altri hanno centrato l'attenzione sulla relazione fra aiuti e investimenti (questi ultimi ritenuti come la causa diretta dei processi di crescita). 
I risultati di questa vasta letteratura sono controversi e danno segnali perlomeno ambigui. In un recente contributo, Rajan e Subramanian (2005) esprimono così l'essenza del dibattito sull'efficacia degli aiuti per la crescita: "one of the most important and intriguing puzzles in economics: why is it so hard to find a robust effect of aid on the long-term growth of poor countries, even those with good policies?"

In questo lavoro ci soffermeremo su due strumenti di politica (politiche di formazione del capitale fisico e umano e aiuti a quelle collegati), in quanto riteniamo siano quelli principali. Essi infatti intervengono sui fattori produttivi di base e lo fanno sulla base di un modello interpretativo teorico chiaro. Le altre politiche possono considerarsi complementari alle precedenti: o come sottolineatura della necessità di un buon contesto macroeconomico (ad es. il contenimento del debito estero), o a livello delle condizioni sociali necessarie perché vengano avviati processi di crescita consistenti (ad esempio il problema della crescita della popolazione, di buone istituzioni e di un clima di fiducia).

Nel lavoro non affronteremo approfonditamente l'impatto delle politiche di liberalizzazione sulla capacità di crescita dei paesi e sulla povertà. La maggioranza degli studi empirici mostra che varie misure di apertura al commercio sono positivamente associate con la crescita (ADES; GLAESER, 1999; ALESINA; SPOLAORE; WACZIARG, 2000; WINTERS, 2004, SPOLAORE; WACZIARG, 2005). Non vi sono casi di paesi che abbiano conseguito rilevanti successi in termini di crescita rimanendo sostanzialmente chiusi ai rapporti economici internazionali (LINDERT; WILLIAMSON, 2003). Vi sono tuttavia alcuni punti critici nella relazione tra politiche di apertura commerciale e crescita che vanno considerati con attenzione. Talune evidenze mostrano che la liberalizzazione degli scambi fa emergere effetti collaterali non desiderabili (RODRIGUEZ; RODRIK, 2001; GALOR; MOUNTFORD, 2006): il commercio internazionale può "bloccare" un paese in specializzazioni che danno pochi vantaggi dinamici, non valorizzare il capitale umano esistente, né incentivarne la formazione).

\section{L'efficacia per la crescita degli aiuti all'investimento in capitale fisico}

John Holsen, un economista della Banca Mondiale, sviluppò nel 1971 un programma per computer il cui obiettivo era stimare il fabbisogno di investimenti di un paese e fornire informazioni sul livello necessario d'aiuti. Si chiamava Minimum Standard Model (MSM). II modello stimava le necessità d'investimento sulla base di un approccio delle interdipendenze settoriali sviluppato da Chenery e Strout (1966). Qualche anno più tardi il modello fu aggiornato e diventò il Revised Minimum Standard Model (RMSM). Il modello continuò ad essere utilizzato per la stima delle necessità di investimento e per fare previsioni sulla crescita nei diversi paesi, anche dopo la diffusione dell'idea che la pura accumulazione di capitale fisico non fosse una condizione sufficiente per lo sviluppo e anche quando divenne chiaro che esso non forniva stime corrette (MEIER, 1995; TODARO, 2000). ${ }^{2}$
2. Vi sono rapporti della Banca Mondiale che si basano su tale approccio per stimare le opportune politiche dopo le crisi macroeconomiche e monetarie in America Latina, in Asia o nel periodo di transizione dal comunismo al capitalismo nei paesi dell'ex blocco sovietico (World Bank, 1995; World Bank, 1997).

Cadernos de Arquitetura e Urbanismo, v.16, n.18+19, 2009 


\section{II rapporto fra aiuti e investimenti}

La verifica del legame empirico fra aiuti e investimenti ha dominato la prima fase degli studi sull'efficacia degli aiuti (GRIFFIN; ENOS, 1970; PAPANEK, 1972; si vedano HANSEN; TARP, 2000; ROODMAN, 2007). Gli aiuti sostengono la crescita (attraverso gli investimenti) se non spiazzano totalmente il risparmio nazionale (i governi che ricevono aiuti possono infatti spostare gli eventuali risparmi ad altre spese di tipo corrente e non ad investimenti. In questo caso l'effetto degli aiuti sugli investimenti e sulla crescita verrebbe a mancare).

Molti studi di letteratura affermano che in effetti in molti casi tale piazzamento esiste, è rilevante, quando non totale, per cui l'aiuto non ha effetto. II modello teorico su cui si basava l'idea dell'efficacia degli aiuti si rivela non consistente nei fatti (vedi BOONE, 1996; EASTERLY, 1999; HARM; LUTZ, 2004; DOUCOULIAGOS; PALDAM, 2006).

\section{II rapporto fra aiuti e crescita}

Un secondo approccio ha cercato di misurare il legame fra tasso di crescita e aiuti, lasciando implicito il passaggio attraverso gli investimenti. Doucouliagos e Paldam (2008) in una meta-analisi dei risultati di regressioni fra crescita e aiuto trovano che fra le 68 migliori regressioni proposte in letteratura, solo il $46 \%$ individua un risultato significativo e positivo nel legame aiuto/crescita. Se si considera l'assai più ampio insieme di regressioni che la letteratura riporta (543 casi) la quota di risultati in cui l'elasticità aiuti/ crescita è positiva e significativa si riduce al $38 \%$. Essi notano inoltre che la varianza fra i diversi coefficienti negli studi empirici condotti, è assai maggiore di quella attesa nel caso di distribuzione casuale dei risultati attorno a un'unica media; i due autori concludono che ciò potrebbe suggerire la presenza di "sottogruppi" di regressioni aventi diverse medie. Ciò sembra rendere ragionevole l'ipotesi di condizionalità: che cioè l'aiuto sia favorevole alla crescita in un contesto che ha determinate caratteristiche.

Radelet, Clemens, Bhavnani (2004) analizzando la vasta letteratura sul rapporto tra aiuti e crescita, classificano i contrastanti risultati nel modo seguente:

- I'aiuto non ha effetti sulla crescita o può persino contrastarla (BAUER, 1972; GRIFFIN; ENOS, 1970; MOSLEY, 1980; MOSLEY et al., 1987; DOWLING; HIEMENZ, 1983; BOONE, 1994). Le ragioni addotte per spiegare tali risultati rimandano agli effetti collaterali degli aiuti nel paese ricevente, dei quali si parlerà nel paragrafo 3 . Alcuni di questi studi, specialmente quelli di più antica data, sono considerati carenti dal punto di vista metodologico. La presenza di fenomeni di endogenità ad esempio è spesso non considerata.

- L'aiuto ha una relazione positiva con la crescita ma mostra rendimenti decrescenti al crescere degli aiuti stessi (HANSEN; TARP, 2001; DALGAARD; HANSEN; TARP, 2004). Si tratta di un approccio che ha come riferimento teorico soggiacente un modello in cui il fattore capitale ha rendimenti decrescenti.

- L'aiuto mostra una relazione condizionale con la crescita e la favorisce solo sotto determinate circostanze, legate in particolare a caratteristiche interne del paese che riceve gli aiuti (ISHAM; 
KAUFMANN; PRITCHETT, 1995; BURNSIDE; DOLLAR 1997), come la qualità delle politiche (BURNSIDE; DOLLAR, 1997), delle istituzioni (BURNSIDE; DOLLAR, 2004), la presenza di forme democratiche di governo (ISLAM, 2003).

\section{Istruzione, capitale umano e crescita}

La seconda grande politica proposta nel dopoguerra per favorire la crescita dei paesi non sviluppati, accanto a quella dell'accumulazione di capitale fisico, è stata quella di sviluppare il capitale umano attraverso massicci programmi di scolarizzazione.

L'importanza del capitale umano parte dal dato che gran parte della crescita nelle economie sviluppate non può essere spiegata dall'aumento quantitativo dei fattori impiegati e che la varianza fra i redditi da lavoro costituisce la causa più importante delle differenze fra i redditi personali. "People with more education have higher wages. This is probably the second (after Engel's law) most well-established fact in economics" (PRITCHETT, 2001, p. 368). E' quasi inevitabile trarre la conseguenza che l'aumento della scolarizzazione, se questa coincide con un aumento dello stock di capitale umano, avrà effetti positivi sulla crescita.

La convinzione che l'istruzione, elemento fondamentale del capitale umano, aumenti la produttività del lavoro e favorisca la crescita, ha fatto della scolarizzazione uno degli assi portanti delle politiche pubbliche per la crescita economica (EASTERLY, 2001a). La Conferenza mondiale sull'educazione del 1990 stabilì che occorreva garantire l'istruzione primaria in tutti i paesi del mondo entro il 2000. II World Development Report 2007 della Banca Mondiale è dedicato all'importanza del capitale umano per il futuro delle giovani generazioni. Tra il 1960 e i primi anni del nuovo millennio si è assistito ad un'esplosione della scolarizzazione, anche grazie all'impulso dato a tale politica dalla Banca Mondiale e da altri organismi internazionali.

L'aumento della scolarizzazione non ha avuto però quel forte impatto in termini di crescita che ci si aspettava. Questa differenza fra aspettative e realtà ha condotto alla rilevante mole di studi empirici sul tema istruzione/crescita e all'identificazione dei problemi ancora irrisolti nella misurazione corretta di tale relazione.

\section{I rendimenti dell'istruzione - l'approccio micro}

L'analisi del capitale umano parte dall'assunto che gli individui decidono del loro livello di istruzione, formazione professionale e altre forme di acquisizione della conoscenza, sulla base di un bilancio fra benefici e costi (BECKER, 1993). I primi includono sia incrementi nelle retribuzioni legate a un maggior livello d'istruzione e conoscenza, sia benefici non monetari (ad esempio culturali, relazionali). I costi sono quelli monetari diretti legati alla frequenza scolastica e i costi opportunità del mancato guadagno durante gli anni spesi in istruzione e in altre forme di acquisizione della conoscenza.

Il concetto di capitale umano è ampio e comprende, oltre all'istruzione "incorporata" nell'individuo, anche esperienze e attitudini acquisite o innate, che possono avere effetti sulla produttività del lavoro dell'individuo e quindi contribuire alle retribuzioni che 
riceve. In tal senso, il concetto di capitale umano è versatile e potente; ciò tuttavia implica che la sua misura non sia immediata né facile. L'istruzione scolastica viene considerata una dimensione decisiva del capitale umano, anche per la facilità della sua quantificazione (gli anni di scuola di un individuo e i risultati scolastici sono osservabili) e una letteratura immensa (vedi per alcune rassegne PSACHAROPOULOS, 1985; PSACHAROPOULOS; PATRINOS, 2002) mostra che i rendimenti in istruzione sono positivi. Tuttavia, non è chiaro se gli anni di scuola (e l'apprendimento nel corso della vita lavorativa) rappresentano in maniera corretta il capitale umano (COHEN; SOTO, 2007). ${ }^{3}$ In effetti, se il capitale umano raccoglie diverse dimensioni e comprende caratteristiche individuali, familiari, relazionali, effetti-paese, ridurre la sua misurazione alla pura quantità di istruzione formale è troppo limitativo. L'incremento di remunerazione (e di produttività) generato dall'istruzione dipende dal contesto in cui il lavoratore si trova ad operare, ad esempio.

\section{Capitale umano e crescita: I'approccio macro}

Dal punto di vista teorico ci sono almeno tre meccanismi attraverso cui l'istruzione (considerata come dimensione rilevante del capitale umano) incide sulla crescita. L'istruzione, aumentando le abilità individuali permette un aumento di produttività e quindi favorisce la transizione a equilibri più elevati di reddito pro capite (MANKIW; ROMER; WEIL, 1992); I'istruzione aumenta la capacità innovativa di un'economia (LUCAS, 1988; ROMER, 1989; AGHION; HOWITT, 1998); I'istruzione facilita la diffusione e la trasmissione di conoscenze necessarie a comprendere i nuovi processi e le nuove tecnologie (NELSON; PHELPS, 1966; BENHABIB; SPIEGEL, 2005). Inoltre, all'accumulazione di capitale umano sono associate esternalità non pecuniarie. L'istruzione in particolare quella femminile - è negativamente correlata con il tasso di natalità (BEHRMAN, 1990) e con la mortalità infantile (BARRO, 1991; BARRO; LEE, 1994; GLEWWE, 2000). La Banca Mondiale riporta che in Africa un incremento del 10\% nel tasso di alfabetizzazione femminile induce una riduzione del $10 \%$ del tasso di mortalità infantile. Il cambiamento nel livello di alfabetizzazione maschile non ha invece effetti in tal senso. Altri effetti positivi dell'istruzione sono la riduzione della criminalità, una partecipazione politica e sociale più consapevole e informata, una maggiore attenzione all'ambiente, una salute migliore e una coesione sociale più ampia. ${ }^{4}$

Tuttavia, i risultati empirici degli studi aggregati sul rapporto fra livello di istruzione e crescita sono contrastanti. Molti studi condotti negli anni Ottanta e all'inizio degli anni Novanta, sottolinearono con entusiasmo l'importanza del capitale umano nella spiegazione del "residuo" nella crescita economica dei paesi occidentali in un contesto teorico di tipo neoclassico. ${ }^{5}$ Un secondo gruppo di studi appare più critico. Lau, Jamison e Louat (1991), in un modello basato su una funzione di produzione Cobb-Douglas applicato a 58 paesi, trovano che l'istruzione ha effetti negativi sulla crescita in Africa, nel Medio Oriente, insignificanti nell'Asia meridionale e in America Latina e positivi solo nell'Asia Orientale. II World Development Report del 1995 sottolinea la poca importanza dell'istruzione nella spiegazione della crescita aggregata
3. II problema era già stato sollevato da Becker nella sua Nobel Prize Lecture nel 1992 (BECKER, 1992).

4. Vedi OECD (1998) per una sintetica rassegna in materia.

5. I primi studi utilizzavano in prevalenza indici di alfabetizzazione adulta (AZARIADIS; DRAZEN, 1990; ROMER, 1990) o tassi di iscrizione scolastica (BARRO, 1991; MANKIW; ROMER; WEIL, 1992; LEVINE; RENELT, 1992) come proxy del capitale umano. Ulteriori tentativi si sono basati su stime degli anni medi di scuola nella popolazione attraverso metodi di inventario perpetuo o simili (LAU; JAMISON; LOUAT, 1991; NEHRU; SWANSON; DUBEY, 1995; confronta per una rassegna WÖSSMANN, 2003). Barro e Lee (1993, 2001) hanno esteso il lavoro proponendo un database comparabile a livello internazionale sugli anni medi di scuola.

Cadernos de Arquitetura e Urbanismo, v.16, n.18+19, 2009 
(World Bank, 1995). I paesi in sviluppo hanno fatto ampie politiche di scolarizzazione senza ottenere tuttavia risultati confortanti in termini di crescita.

\section{La qualità dell'istruzione}

L'indeterminatezza dei risultati delle analisi macro sugli effetti dell'istruzione ha condotto alla ricerca dei fattori che possono favorire o bloccare gli effetti dell'istruzione sull'evoluzione del prodotto aggregato. Pritchett (2001), ad esempio, ritiene che le cause della paradossale diversità fra risultati delle analisi micro e quelli delle analisi macro siano legate a specifiche condizioni dei paesi in via di sviluppo: il sistema scolastico e la sua qualità e il mercato del lavoro.

Per quanto riguarda il sistema scolastico, è sotto accusa la qualità dell'istruzione che può essere così bassa da non incrementare le abilità e la produttività dell'individuo. Hanushek e Wössmann (2007) affermano che "educational quality, particularly in assessing policies related to developing countries, is THE key issue." In un'analisi cross-country Hanushek e Kimko (2000) evidenziano che l'aggiunta di indici qualitativi a quelli quantitativi fa passare la varianza spiegata nei PIL pro capite dei diversi paesi dal 33\% al 73\%. Questo risultato è proprio di molti altri studi, in cui si evidenzia che I'aspetto della qualità è dominante (BOSWORTH; COLLINS, 2003; CICCONE; PAPAIOANNOU, 2005). Mentre le politiche di scolarizzazione hanno portato ad un incremento quantitativo di questa, la qualità dell'istruzione - in molti paesi in sviluppo - non solo non è migliorata, ma in molti casi è persino diminuita.

Per quanto riguarda il mercato del lavoro, l'incremento dell'offerta di forza lavoro istruita in presenza di domanda stagnante può causare una riduzione delle relative retribuzioni; di conseguenza l'aumento del PIL sarà minore di quello che si poteva prevedere sulla base dei rendimenti attesi. La domanda di lavoro istruito dipende da caratteristiche del sistema produttivo. In questo caso possiamo possono esistere rendimenti positivi a livello delle retribuzioni individuali ma un impatto nullo a livello macroeconomico, in quanto tali differenze rappresentano solo una redistribuzione di reddito tra gli individui. Ad esempio, in molti paesi in via di sviluppo il settore pubblico assorbe la crescente offerta di forza lavoro istruita, rispondendo a pressioni politiche (GELB; KNIGHT; SABOT, 1991). In Egitto nel 1998 il settore pubblico occupava il 70\% di tutti i laureati e il $63 \%$ dei lavoratori con livelli medi di istruzione.

\section{Le cause dell'inefficacia degli aiuti e la condizionalità}

Le politiche di aiuto che avrebbero dovuto generare crescita sono fallite per non aver saputo generare opportuni incentivi al comportamento degli agenti; spesso anzi hanno generato incentivi distorti. Qui di seguito sono analizzate sinteticamente le principali cause che vi hanno condotto.

\section{Complementarietà ed esternalità}

Se il cambiamento tecnologico è la determinante principale del livello del reddito, perché i paesi poveri non hanno adottato 
tecnologie avanzate? L'arretratezza tecnologica può essere un vantaggio perché permette di saltare direttamente alla frontiera tecnologica, grazie al processo d'imitazione e all'afflusso di investimenti diretti dall'estero, veicolo privilegiato per la diffusione di conoscenze tecnologiche (BORENSZTEIN, de GREGORIO; LEE, 1998; BLOMSTRÖM; LIPSEY; ZEJAN, 1994). Tale potenziale vantaggio tuttavia può mutarsi in uno svantaggio, se la capacità di usare le nuove tecnologie dipende da una certa omogeneità nei livelli tecnologici dei diversi settori e nel know how necessario per un loro efficiente utilizzo (in altri termini, se esistono forme di complementarità e di indivisibilità). Gli individui accumulano competenze (skills) dove ci sono tecnologie avanzate, gli imprenditori investono in nuove tecnologie dove ci sono lavoratori qualificati. La complementarità tra tecnologia e lavoro qualificato genera anche una complementarità tra lavoratori: la produttività di ciascun lavoratore non dipende solo dalla propria qualificazione e competenza ma anche da quella degli altri (matching). ${ }^{6}$ Dato che la diffusione delle conoscenze e la cattura dei rendimenti sociali delle stesse è legata all'interazione fra i diversi attori (complementarità), possono verificarsi coordination failures. Questi, se diffusi, possono generare equilibri di basso livello (poverty traps; si veda HOFF, 2000). ${ }^{7}$ L'esistenza di esternalità e di coordination failures possono spiegare perché gli investimenti in capitale fisi$\mathrm{co} e$ in capitale umano tendono ad affluire verso i paesi più ricchi di conoscenze che offrono rendimenti più alti (ACEMOGLU, 1997). Mankiw (1995) osserva ad esempio che l'assenza di flussi di capitale verso paesi in cui è assente il lavoro qualificato può essere collegata al fatto che, di conseguenza, i rendimenti del capitale fisso sono bassi. Tuttavia va notato che nei paesi in via di sviluppo una maggiore educazione è associata molto spesso alla disoccupazione, per mancanza di domanda di forza lavoro skilled (KRUEGER; LINDHAL, 2001; AL-SAMARRAI; BENNELL, 2007). Vi sono comunque casi nei quali il coordinamento fra le diverse dimensioni ha avuto successo. Lo sviluppo del capitale umano nell'Asia dell'Est, per esempio, ha permesso a queste economie di acquisire e sfruttare le conoscenze tecnologiche, le nuove idee, i nuovi processi produttivi ed ha cosi permesso di raggiungere un'alta produttività (World Bank, 1991). Benhabib e Spiegel (1994) trovano evidenze di esternalità positive dell'accumulazione di capitale umano nel facilitare l'adozione di nuove tecnologie.

Un'altra fonte di poverty traps riguarda le esternalità associate al comportamento degli agenti e delle istituzioni. Un famoso lavoro di Murphy, Schleifer e Vishny (1993) mette in evidenza che condizioni istituzionali che rendono possibile l'attività di rent seeking, ad esempio la possibilità di imporre tasse (formali e informali) sull'attività agricola rivolta al mercato e all'esportazione, possono condurre i produttori delle aree rurali a scegliere di mantenere coltivazioni legate alla pura sussistenza, in sé meno redditizie. Infine, può essere razionale per un paese "accettare" di rimanere arretrato (rational underdevelopment: DESMET; ORTÍN, 2007) se i frutti del progresso tecnologico vengono acquisiti soprattutto dalle economie già avanzate, un paese arretrato, può decidere di scegliere la condizione di arretratezza in cambio di sussidi e di trasferimenti da parte delle economie più sviluppate (forme di dualismo).
6. Esternalità simili a quelle legate agli investimenti in istruzione si verificano anche per gli investimenti in attività di ricerca e sviluppo delle imprese: se i rendimenti degli investimenti in $R \& S$ per la singola impresa dipendono dal numero di imprese che investono in $R \& S$, cioè dal pool complessivo di idee esistenti e pubblicamente disponibili, nessuna impresa avrà incentivo ad investire quando il numero delle imprese innovatrici è basso. Si può generare così una situazione di equilibrio di basso livello.

7. I problemi di coordinamento generati dalla presenza di imperfezioni di mercato, nella tradizione di Coase potrebbero venir risolti attraverso forme di contrattazione che specificano i diritti di proprietà sui diversi effetti, attraverso forme di acquisizione e di fusione fra imprese i cui processi mostrano interdipendenze, ecc. Tuttavia, quando queste interdipendenze ed esternalità sono diffuse, un simile approccio non risolve i problemi.

Cadernos de Arquitetura e Urbanismo, v.16, n.18+19, 2009 


\section{Governi, politiche e assetto istituzionale}

Politiche governative non appropriate possono impedire la crescita. II mantenimento di alti tassi di inflazione, una rilevante differenza fra tassi di cambio ufficiali e quelli di mercato nero (black market premium), tassi d'interesse reali negativi, elevati deficit nei bilanci pubblici, forme di restrizione al libero commercio, eccessiva burocrazia e servizi pubblici inadeguati, ne sono degli esempi.

La crescita è ostacolata se i governi sono incapaci di fornire servizi pubblici di qualità: energia elettrica, linee telefoniche, strade, ospedali, I'acqua, le fognature, l'irrigazione, servizi postali, servizi sanitari di base, discariche per i rifiuti. In Uganda, secondo dati del periodo 1995-1997, la fornitura d'acqua subiva 33 giorni di interruzione all'anno, il $77 \%$ delle imprese aveva discariche private per i rifiuti e solo il $31 \%$ della corrispondenza d'affari veniva spedita dall'ufficio postale (REINIKKA; SVENSSON, 1999).

La corruzione è presente in tutti i paesi a livelli diversi. Le analisi empiriche mostrano che la corruzione ha effetti negativi - sia diretti che indiretti - sulla crescita. Che cosa sia corruzione e come definirla è una questione complessa. Anche dal solo punto di vista economico, esistono diverse accezioni e una complessa tassonomia dei fenomeni di corruzione (si veda ad esempio la rassegna di Bardhan, 1997). Hopkin (2002) ritiene che le basi esplicative della corruzione siano pre-economiche (citando Schumpeter, 1961, secondo cui esiste un "non economic bottom"). Bardhan (1997) sottolinea che ragioni temporanee che aumentano la corruzione (un conflitto interno, un disastro ambientale che coinvolge la nazione) possono avere effetti permanenti. La reputazione collettiva, una volta compromessa, è difficile da ricostruire.

Fattori che influenzano il livello di corruzione di un paese sono la differenziazione etnica, il black market premium, le restrizioni al libero commercio e la qualità delle istituzioni. Una maggiore differenziazione etnica, può aumentare la probabilità di corruzione perché le diverse parti cercheranno di appropriarsi di una quota delle risorse comuni (SVENSSON, 2000). Se c'è un mercato nero della valuta, ogni funzionario in possesso di una licenza per l'acquisto di dollari al tasso di cambio ufficiale può fare profitti rivendendo i dollari al mercato nero ed è quindi incentivato alla corruzione. Lo stesso avviene in presenza di limitazioni legali commercio internazionale (ADES; DITELLA, 1999). La mancanza di rispetto dei contratti da parte del governo rende più probabile la corruzione, perché i privati cittadini dovranno corrompere i funzionari statali per garantirsi il rispetto dei loro contratti. Le discrezionalità nelle forme di esproprio, ad esempio, sono decisive nelle relazioni imprese-governo. Se vi è un alto rischio di esproprio, gli imprenditori pagheranno, pur di non subirlo. In generale quindi vi è una forte associazione tra qualità istituzionale $e$ corruzione, anche se occorre avere cautela nel definire i nessi di causalità: pessime politiche da parte del governo possono essere causate sia dalla corruzione sia da pessime istituzioni (KNACK; KEEFER, 1995).

Come impedire la corruzione? Secondo alcuni (ROSE-ACKERMAN, 1999) occorre semplificare i sistemi fiscali e amministrativi, 
eliminare i sussidi statali; introdurre la competizione fra differenti agenzie governative per la fornitura dello stesso servizio. Molte di queste azioni richiedono un' "autorità" in grado di instaurare meccanismi di monitoraggio e sanzione credibili; tuttavia in sistemi con corruzione endemica, anche tale istituzione non sfuggirebbe facilmente al gioco. La proposta più interessante sembra quella di istituire una molteplicità di agenzie pubbliche che possono (in competizione) fornire gli stessi servizi. Ad esempio negli Stati Uniti il coinvolgimento sovrapposto fra polizia locale, statale e federale ha ridotto la corruzione degli agenti nel controllo del traffico di droga. Questo però può condurre anche a forme di lassismo nelle amministrazioni.

Una caratteristica che incide negativamente su comportamenti coerenti con la crescita, è la presenza di forme di polarizzazio-

ne e di frazionamento del tessuto sociale. In società divise si hanno incentivi a redistribuire il reddito esistente, piuttosto che a promuovere lo sviluppo. La polarizzazione sociale aumenta la possibilità di conflitti interni, è causa di cattive politiche governative e di sovrasfruttamento delle risorse comuni. Un aspetto della polarizzazione sociale è la presenza di forti disuguaglianze fra i redditi dei diversi gruppi, e c'è evidenza che paesi con più forte disuguaglianza sono politicamente più instabili, più soggetti a rivoluzioni e a colpi di stato (EASTERLY, 2000; 2001b).

\section{La condizionalità come strumento correttivo}

La constatazione che cattive politiche e comportamenti scorretti da parte dei governi sono nocivi alla capacità di crescita, suggerì alle istituzioni internazionali e ai donors, che occorreva orientare i paesi verso pratiche di "buon governo" con interventi che almeno parzialmente incidevano sulla loro sovranità. L'intervento da parte di istituzioni internazionali nel modo di agire degli Stati è il punto di arrivo di un'evoluzione avvenuta nel corso del dopoguerra. Benché le Nazioni Unite, fossero già un'esperienza di coordinamento sovranazionale, alla fine del secondo conflitto mondiale i singoli Stati, in particolare i Paesi di nuova indipendenza, erano gelosi della propria sovranità. Anche la contrapposizione tra i due blocchi della Nato e del Patto di Varsavia contribuiva ad impedire a qualunque istituzione internazionale di intervenire con forza nelle politiche interne degli Stati. Tuttavia, dagli anni Settanta, le Istituzioni Finanziarie Internazionali (IFI) cominciarono a suggerire ai paesi in difficoltà le misure di disciplina monetaria e fiscale (a breve termine) e la ristrutturazione del ruolo dello stato nei confronti dei mercati (a lungo termine) ritenute necessarie. Iniziò così una lenta erosione della piena sovranità degli Stati, i quali, almeno per quanto riguarda la dimensione economica, si trovarono a dover dialogare con istanze di tipo superiore. A quel tempo esisteva (WEISS, 2000) una contrapposizione fra Washington (sede delle istituzioni finanziarie) dove si sottolineava che occorrevano cambiamenti nello stile di governo di molti Stati, e New York (sede delle Nazioni Unite) dove invece si resisteva a simili ingerenze. II sistema di voto per paese vigente presso le Nazioni Unite, portava i paesi emergenti a "difendere" con successo la propria sovranità da ingerenze esterne. Col passare del tempo, tuttavia, la convinzione che qualche forma d'intervento fosse necessaria, conquistò anche le Nazioni Unite. In effetti, i paesi non 
occidentali avevano fatto pressioni (alla fine vincenti) perché le istituzioni internazionali sanzionassero governi retti da minoranze bianche che attuavano forme di apartheid (Sudafrica, Rhodesia); a questo punto era impensabile impedire interventi internazionali di denuncia verso regimi come quello di Idi Amin in Uganda, di Pol Pot in Cambogia, o di Duvallier ad Haiti. Tale passaggio risulterà decisivo: nei decenni precedenti le IFI avevano insistito sul fatto che occorrevano buone politiche economiche; negli anni Ottanta la comunità internazionale iniziò ad affermare anche la necessità di "un buon sistema politico". Gli interventi umanitari in occasione di conflitti estesero ulteriormente l'intervento di forze internazionali nella vita interna degli Stati. Come sinteticamente disse I'allora Segretario delle Nazioni Unite, Boutros-Ghali, "the time of absolute and exclusive sovereignty, however, has passed" (BOUTROS-GHALI, 1992). II passaggio da quello che è stato chiamato Washington Consensus (WILLIAMSON, 1990) all'Augmented Washington Consensus segna esattamente questo percorso. La prima "lista" che componeva il Washington Consensus era sostanzialmente un insieme di richieste relative a politiche economiche. II Washington Consensus "aumentato" aggiunge invece una serie di richieste che riguardano il comportamento di governi e stati, la qualità delle istituzioni e gli obiettivi ultimi delle politiche economico-sociali.

Uno strumento per orientare i paesi verso riforme politiche fu la concessione di prestiti condizionati. La prima generazione di tali prestiti fu collegata al forte indebitamento estero di alcuni paesi, alle conseguenti crisi debitorie verso banche estere e istituzioni internazionali, agli alti tassi d'inflazione e alle svalutazioni. Le istituzioni internazionali decisero di legare gli aiuti (in questo caso per la gestione del debito) a riforme di politica economica (prestiti di aggiustamento strutturale). Tali prestiti erano spesso indirizzati a paesi con situazioni di partenza difficili, alta inflazione, deficit di bilancio, alto black market premium, tassi d'interesse negativi. Tra il 1980 e il 1994, lo Zambia ricevette dodici prestiti d'aggiustamento, ma in gran parte dello stesso periodo il paese mantenne un'inflazione a due cifre. Sebbene la diminuzione dell'inflazione fosse una delle condizioni per ricevere i prestiti, l'inflazione continuò a crescere assieme agli aiuti. In molti casi, la condizione di non concedere prestiti a paesi che mantenevano alti deficit di bilancio o che mantenevano tassi d'interesse reali negativi, non fu rispettata. Tra il 1980 e il 1994 la Banca Mondiale e il FMI concessero alla Costa d'Avorio diciotto prestiti d'aggiustamento, nonostante un deficit di bilancio pari al $14 \%$ del PIL. II tasso d'interesse reale era negativo in molti paesi che ricevettero sostanziosi prestiti degli organismi internazionali. II Nicaragua, ad esempio, tra il 1989 e il 1991 ebbe un tasso d'interesse reale negativo pari a $-86,7 \%$ e ricevette aiuti ufficiali allo sviluppo pari al 54,5\% del PIL.

Prestiti di aggiustamento sono stati spesso accordati a governi corrotti. Un governo corrotto ha incentivi a rimanere tale, una volta ottenuti i prestiti; e perciò è improbabile che ci siano sostanziali miglioramenti nella politica economica. Molti governi hanno scelto di ridurre il deficit (necessario per ottenere gli aiuti) attraverso interventi di breve periodo: tagliando gli investimenti in infrastrutture o svendendo imprese statali, chiedendo versamenti anticipati delle tasse, oppure facendosi sovvenzionare dai 
fondi pensione. Sono misure che riducono il deficit attuale, permettendo quindi di rispettare le condizioni legate ai prestiti, ma rinviano solo il problema ai periodi successivi. Sono critiche alla politica degli aiuti condizionati che insistono sul fatto che è difficile far rispettare le condizioni poste, in sé buone.

Una posizione differente è quella che giudica inefficaci gli aiuti condizionali perché le politiche proposte sono in sé sbagliate e non conducono alla crescita. Questa seconda posizione è ad esempio sostenuta da Rodrik (2007). Egli sottolinea come le economie che hanno avuto le migliori performance sono quelle che non hanno seguito l'ortodossia delle riforme strutturali. Cina e Vietnam, ad esempio hanno attuato una politica del "doppio binario" (liberalizzazione in certi settori, pianificazione centralizzata in altri), non hanno seguito le regole "commerciali" proposte dal GATT e dal WTO; I'India, ha attuato le riforme in maniera lenta e graduale. Dall'altro lato, molti paesi latino-americani che hanno adottato l'agenda "standard" di riforme, hanno avuto esiti meno buoni o negativi. Rodrik conclude che la capacità di suggerire exante quali riforme adottare è limitata e che dare consigli basati su una lista di "giuste" riforme può condurre a risultati opposti. La sua proposta è realizzare caso per caso un'approfondita analisi diagnostica, per esaminare gli effetti delle riforme proposte e la presenza di specifici vincoli alla crescita, e disegnare - sulla base della stessa - l'opportuno impianto di politiche. Sia il processo di diagnosi che il disegno delle politiche deve però essere istituzionalizzato per garantirne la durata nel tempo.

\section{Conclusioni}

La rassegna della letteratura sull'efficacia delle due proposte di politica per la crescita più condivise e applicate (l'aiuto agli investimenti in capitale fisico e umano) ha reso evidente che non si è ancora giunti a risultati chiari e robusti. Negli ultimi anni si è di conseguenza intensificato il tentativo di comprendere quali vie seguire per riguadagnare efficacia nelle attività di aiuto, problema decisivo sia per le istituzioni internazionali preposte al sistema degli aiuti sia per gli altri donors.

\section{Complessità e specificità}

La relazione aiuti/investimenti/crescita è molto più complessa di quanto ci si aspettasse: i nessi fra aiuto e investimento e fra investimento e crescita dipendono da numerosi elementi di contesto, spesso ancora difficili da classificare e da valutare, sul piano teorico e nella verifica empirica. Tale complessità e la constatazione delle numerose interdipendenze fra diverse dimensioni implicate nella catena di relazioni descritta, hanno condotto, ormai da una decina d'anni, a riproporre modelli e approcci del tipo big push, recuperando un'idea originale di Rosenstein-Rodan (vedi HOFF, 2000; EASTERLY, 2005; BOWLES; DURLAUF; HOFF, 2006).

D'altra parte, complessità e multidimensionalità (economica, sociale e istituzionale) richiedono strategie di azione più attente alla specificità delle situazioni e dei contesti. Nella prefazione al rapporto del 2005 "Economic growth in the 1990s - learning from a decade of reforms", preparato dal Poverty Reduction and 
Economic Management (PREM) Network della Banca Mondiale (World Bank, 2005), si riconosce che l'approccio delle istituzioni internazionali alle politiche di crescita è stato nei decenni scorsi schematico, rigido e incapace di individuare gli opportuni sentieri di crescita. Viene affermato che "the central message (...) is that there is no unique universal set of rules (...) we need to get away from formulae and the search for elusive 'best practices' and rely on deeper economic analysis to identify the binding constraints on growth" (p. xiii). Al tema della country specificity (strategie di crescita "disegnate" sulla condizione del paese) si associa quello della country ownership nella gestione delle politiche stesse. Si tratta di obiettivi e di linee guida per l'azione proposte nella Dichiarazione di Parigi del 2005 sull'efficacia degli aiuti, e riprese con forza nel recente incontro di Accra (vedi la Accra Agenda for Action).

\section{Grandi piani, azione individuale e sussidiarietà}

La necessità di un approccio country specific gestito dagli attori locali è condivisa da molti. Assai diverse sono invece le opinioni su come tradurre in pratica questo nuovo approccio. Per alcuni complessità e specificità possono essere affrontate solo attraverso piani globali e comprensivi che "controllano" in modo simultaneo le differenti dimensioni della crescita e dello sviluppo (Easterly, 2008 chiama tale approccio "transformational").

Solo gli Stati, allora, sono nella posizione di poterli gestire: questi divengono il partner decisivo, nelle attività di cooperazione internazionale. E' una direzione giusta o sbagliata?

A nostro avviso ci sono due errori in questa posizione. II primo è che quanto più una politica è complessa e filtrata dal sistema di governo e amministrativo, tanto più è soggetta al rischio che i diversi attori (in primo luogo quelli pubblici) assumano comportamenti sbagliati, decidano non secondo criteri di bene comune, ma per favorire elite o ceti specifici. La complessità, rende difficile stabilire rapporti di causa ed effetto fra input e risultati: è perciò una tentazione a "firmare" contratti impegnativi, perché un governo che non ha intenzione di onorarli può addurre mille motivi a propria scusa.

Anche la recente politica di budget support attuata dall'Unione Europea, che ha come cardine il sostegno al bilancio degli Stati beneficiari, perché attuino politiche concertate, condivise e monitorate nei risultati per evitare comportamenti sleali, può correre lo stesso pericolo. ${ }^{8} \mathrm{Si}$ ha efficacia nello sviluppo quando persone e gruppi sociali hanno esperienza concreta delle opportunità: le famiglie mandano i figli a scuola (sacrificando un potenziale apporto dei ragazzi al reddito familiare) non per una politica governativa, ma per un'esperienza che ne vale la pena e che è possibile farlo (e in questo le politiche possono aiutare).

II secondo errore è la mancanza di sussidiarietà. Le persone, i gruppi sociali, la società civile non vengono coinvolti. Nella politica dei grandi piani, I'esperienza che la gente fa è che se qualche vantaggio può arrivare è grazie allo Stato: ne consegue un atteggiamento di dipendenza e rivendicativo che non mette in moto la gente, le persone e le realtà sociali.
8. na critica in tal senso alle politiche di budget support è già stata fatta. Si veda al riguardo Lavergne e Wood (2006).

Cadernos de Arquitetura e Urbanismo, v.16, n.18+19, 2009 


\section{Educazione dell'io, fattore dello sviluppo}

Si possono gestire dei piani integrati di crescita e sviluppo coinvolgendo la società civile? Occorre sicuramente il dialogo

dei governi con la società. Questa posizione si impara per esperienza come termine di un cammino educativo dei governi e delle amministrazioni ; occorre inoltre un equilibrio di strumenti e di potere fra governo e società civile. Non basta il "controllo" dei donors sull'uso delle risorse, anche se esso può essere utile, occorre una effettiva corresponsabilità fra dimensione politica e società civile, che è l'essenza della democrazia. $\mathrm{E}^{\prime}$ il principio della partnership.

Tuttavia, anche queste due "regole" di comportamento, da sole non sono sufficienti, sarebbero "sospese sul vuoto", se la società civile non è composta di persone consapevoli delle opportunità, mosse e in cammino. Che cosa lo permette? In un articolo apparso sul Financial Times lo scorso maggio, ${ }^{9}$ William Easterly critica aspramente il paradigma dello sviluppo basato sui grandi piani che, ritiene, porta a danni enormi e a ingenti sprechi e afferma che I'unica possibilità per uno sviluppo effettivo è lasciare all'intraprendenza dei molti (quelli che Easterly chiama i "searchers") di trovare la strada per un uso efficiente delle risorse. Easterly sottolinea provocatoriamente che vi sono al mondo 7 miliardi di esperti (i singoli individui) a cui bisogna lasciare libertà d'azione. Gli Stati, invece di fare grandi piani, dovrebbero garantire lo spazio per l'iniziativa. Questa posizione liberista evidenzia degli aspetti interessanti, ma è parziale. I comportamenti dei singoli, come si è accennato nell'esempio dei contadini, a causa di un contesto non favorevole, possono essere bloccati in condizioni di povertà, quando non si ha esperienza di un altro più opportuno cammino. I movimenti dei Senza Terra in Brasile, ad esempio, restano spesso rivendicativi e dipendenti dal potere politico, se non c'è l'esperienza (per un incontro, per la testimonianza di amici e conoscenti) che vi sono altre vie, più libere, più responsabilizzanti (anche per certi aspetti più faticose). Questa esperienza è il costituirsi del soggetto dello sviluppo.

Anche un approccio di tipo partecipativo può essere altrettanto parziale. Se l'approccio liberista ritiene l'individuo già "formato" e pronto a reagire agli stimoli del reale, la tradizione partecipativa fa lo stesso con la comunità, ritenendola soggetto che su auto-conosce. E' vero che ogni comunità umana, con le sue esperienze e la sua cultura, può sapere meglio di altri ciò di cui ha bisogno e ciò che sente consono al proprio cammino; tuttavia, come sa chi opera in attività di cooperazione internazionale, questa conoscenza locale può essere "binding", limitante e a volte carica di comportamenti perversi, mentre può arricchirsi e inverarsi proprio nell'incontro con altri soggetti e nell'esperienza di cui questi sono tramite. In un simile incontro, bisogni ed esigenze possono ritrovarsi definite meglio di quanto individui e gruppi sappiano fare da soli, perché sfidate e spalancate a una nuova ipotesi e a un assetto più adeguato di fronte ai dati di realtà.

E' I'idea di educazione, una introduzione più piena e profonda alla realtà nel suo significato e nel suo valore per il cammino
9. W. Easterly, "Trust the development experts - all 7bn", Financial Times, May 28,2008 
umano. Sia per le comunità aiutate (da una politica o da un progetto), sia per chi attua le attività di aiuto è l'avventura di una introduzione più adeguata al reale e al suo significato. E' I'educazione - non la semplice scolarizzazione - il motore dello sviluppo.

Mi ha sempre colpito il fatto che chi opera in progetti di sviluppo, in un centro di nutrizione ad esempio, se è intelligente ed "educato" è attento non solo a curare i piccoli (pesarli, stabilire una dieta, fornire il necessario per la stessa) ma soprattutto a dialogare con le madri perché nel cambiamento del modo di vivere e di guardare la realtà delle madri (modo di abitare, igiene, rapporti con altre madri) il bisogno che esse hanno - curare i figli - si chiarisce e diventa più adeguato. E' un cammino educativo e di conoscenza se chi dialoga con loro sente realmente simpatia per il loro cammino e lo condivide "a tutto campo". Come afferma il "principio" bellissimo e vero che ha sempre guidato I'attività dell'AVSI: occorre condividere a tutto campo, fino al senso della vita, perché la condivisione del bisogno sia reale e concreta e generi una mossa stabile di cambiamento, cioè sviluppo.

\section{Riferimenti}

ACEMOGLU, D. Training and innovation in an imperfect labour market. Review of Economic Studies, v. 64, n. 220, p. 445-464, 1997.

ADES, A. F; DI TELLA, R. Rents, competitions, and corruption. American Economic Review, v. 89, n. 4, p. 982-993, 1999.

ADES, A. F.; GLAESER, E. L. Evidence on growth, increasing returns and the extent of the market. The Quarterly Journal of Economics, v. 114, n. 3, p. 1025-1045, 1999.

AGHION, P.; HOWITT, P. Endogenous growth theory. Cambridge: MIT Press, 1998.

ALESINA, A.; SPOLAORE, E.; WACZIARG, R. Economic integration and political disintegration. American Economic Review, v. 90, n. 5, p. 1276-1296, 2000.

AL-SAMARRAI, S.; BENNELL, P. Where has all the education gone in sub-Saharan Africa? Employment and other outcomes among secondary school and university leavers. The Journal of Development Studies, v. 43, n. 7, p. 1270-1300, 2007.

ARROW, K. J. The economic implications of learning by doing. The Review of Economic Studies, v. 29, n. 3, p. 155-173 June 1962.

AZARIADIS, C.; DRAZEN, A. Threshold externalities in economic development. Quarterly Journal of Economics, v. 105, n. 2, p. 501-526, 1990.

BARDHAN, P. Corruption and development: a review of issues. Journal of Economic Literature, v. 35, p. 1320-1346, Sept. 1997.

BARRO, R. Economic growth in a cross section of countries. Quarterly Journal of Economics, v. 106, n. 2, p. 407-443, 1991. 
BARRO, R.; LEE, J-W. International data on educational attainment: updates and implications. Oxford Economic Papers, v. 53, n. 3, p 541-63, July 2001.

BARRO, R.; LEE, J-W. Sources of economic growth. CarnegieRochester Conference Series on Public Policy, v. 40, n. 1, p 1-46, June 1994.

BAUER, P. T. Dissent on development: studies and debates in development economics. Cambridge: Harvard University Press, 1972.

BECKER, G. S. Human capital: a theoretical and empirical analysis, with special reference to education. Chicago: University of Chicago Press, 1993.

BECKER, G. S. The economic way of looking at life: Nobel lecture, Stockholm: The Nobel Foundation, 1992.

BEHRMAN, J. R. Women's schooling and nonmarket productivity: a survey and a reappraisal. Pennsylvania: University of Pennsylvania, 1990.

BENHABIB, J.; SPIEGEL, M. Human capital and technology diffusion. In: AGHION, P.; DURLAUF, S. N. (Ed.). Handbook of economic growth. Amsterdam: North Holland, 2005. p. 935-966.

BENHABIB, J.; SPIEGEL, M. Role of human capital in economic development: evidence from aggregate cross-country data. Journal of Monetary Economics, v. 34, p. 143-173, Oct. 1994.

BLOMSTRÖM, M.; LIPSEY, R. E.; ZEJAN, M. What explains the growth of development countries? In: BAUMOL, W.; NELSON, R.; WOLFF, E. (Ed.). Convergence of productivity: cross-national studies and historical evidence. Oxford: Oxford University Press, 1994.

BOONE, P. Politics and the effectiveness of foreign aid. European Economic Review, v. 40, n. 2, p. 289-329, Feb. 1996.

$B O O N E, P$. The impact of foreign aid on savings and growth. London: London School of Economics, 1994.

BORENSZTEIN, E.; DE GREGÓRIO, J.; LEE, J-W. How does foreign direct investment affect growth? Journal of International Economics, v. 45, p. 115-135, June 1998.

BOSWORTH, B. P.; COLLINS, S. M. The empirics of growth: an update. Brookings Papers on Economic Activity, n. 2, p. 113206, 2003.

BOUTROS-GHALI, B. An agenda for peace - preventive diplomacy, peacemaking, and peace-keeping. Report of the Secretary-General, 1995. Disponível em: <http://www.un.org/ Docs/ SG/agpeace.html>

BOWLES, S.; DURLAUF, S.; HOFF, K. Introduction. In: BOWLES, S.; DURLAUF, S.; HOFF, K. (Ed.). Poverty traps. Princeton: Princeton and Russell Sage Foundation, 2006.

BURNSIDE, C.; DOLLAR, D. Aid, policies, and growth. Washington: World Bank, 1997. (Policy Research Working Paper, n. 1777).

BURNSIDE, C.; DOLLAR, D. Aid, policies, and growth: revisiting the evidence. Washington: World Bank, 2004. (Policy Research Working Paper, n. 3251). 
Returns to investment in human capital. In: CENTRE FOR EDUCATIONAL RESEARCH AND INNOVATION. Human capital investment; an international comparison. Paris: OECD, 1998.

CHENERY, H. B.; STROUT, A. M. Foreign assistance and economic development. American Economic Review, v. 56, n. 4, part I, Sept. 1966.

CICCONE, A.; PAPAIOANNOU, E. Human capital, the structure of production, and growth. London: CEPR, 2005. (CEPR, Discussion Paper n. 5354).

DALGAARD, C-J.; HANSEN, H.; TARP, F. On the empirics of foreign aid and growth. Economic Journal, v. 114, n. 496, p. 191-216, 2004.

DESMET, K.; ORTíN, I. O. Rational underdevelopment. Scandinavian Journal of Economics, v. 109, n. 1, p. 1-24, 2007.

DOUCOULIAGOS, H.; PALDAM, M. Aid effectiveness on growth: a meta study. European Journal of Political Economy, v.24, p. 1-24, 2008.

DOUCOULIAGOS, H.; PALDAM, M. Conditional aid effectiveness: a meta study. Denmark: University of Aarhus, 2005. (Working Paper N. 2005-14).

DOUCOULIAGOS, H.; PALDAM, M. The aid effectiveness literature: the sad result of 40 years of research. Denmark: University of Aarhus, 2005. (Working Paper, n. 2005-15).

DOWLING, J. M.; HIEMENZ, U. Aid savings, and growth in the Asian region. The Developing Economies, v. 21, n. 1, p. 1-13, 1983.

EASTERLY, W. Lo sviluppo inafferrabile: I'avventurosa ricerca della crescita economica nel Sud del mondo. Milano: Bruno Mondadori, 2006.

EASTERLY, W. Reliving the '50s: the big push, poverty traps, and takeoffs in economic development. Washington DC: Center for Global Development, 2005. (Working Paper, 65).

EASTERLY, W. The elusive quest of growth, economists: adventures and misadventures in the tropics. Cambridge: MIT Press, 2001 a.

EASTERLY, W. The ghost of financing gap: testing the growth model of the international financial institutions. Journal of Development Economics, v. 60, n. 2, p. 423-438, Dec. 1999.

EASTERLY, W. The lost decades: explaining developing country stagnation 1980-1998. Journal of Economic Growth, v. 6, n. 2, p. 135-157, $2001 \mathrm{~b}$.

EASTERLY, W. The middle-class consensus and economic development. Washington: World Bank, 2000. (Policy Research Working Paper, n. 2346).

GALOR, O.; MOUNTFORD, A. Trade and the great divergence: the family connection. London: American Economic Association, 2006. (CEPR Discussion Paper, 5490).

GELB, A.; KNIGHT, J. B.; SABOT, R. H. Public sector employment, rent seeking and economic growth. Economic Journal, v. 101, n. 408, p. 1186-99, 1991. 
GLEWWE, P. Schools, skills and economic development: evidence, gaps and research prospects. Washington: World Bank, 2000.

GOLDIN, I.; ROGERS, H.; STERN, N. The role and effectiveness of development assistance: lessons from World Bank experience. Washington: World Bank, 2002. (World Bank Research Paper).

GRIFFIN, K. B.; ENOS, J. L. Foreign assistance: objectives and consequences. Economic Development and Cultural Change, v. 18, n. 3, p. 313-27, 1970.

HANSEN, H.; TARP, F. Aid and growth regressions. Journal of Development Economics, v. 64, p. 547-570, 2001.

HANSEN, H.; TARP, F. Aid effectiveness disputed. Journal of International Development, v. 12, p. 375-398, 2000.

HANSON, G. H. Should countries promote foreign direct investment? G-24 Discussion Paper, n. 9, p. 1-30, 2001.

HANUSHEK, E. A.; KIMKO, D. D. Schooling, labor force quality, and the growth of nations. American Economic Review, v. 90, n. 5, p. 1184-1208, Dec. 2000.

HANUSHEK, E. A.; WÖßMANN, L. The role of education quality in economic growth. Washington: World Bank, 2007. (Policy Research Working Paper, n. 4122).

HARMS, P.; LUTZ, M. The macroeconomic effects of foreign aid: a survey. St. Gallen (Switzerland): University of St. Gallen, 2004. (Discussion Paper, n. 11).

HOFF, K. Beyond Rosenstein-Rodan: the modern theory of underdevelopment traps. Washington DC: World Bank Development Economics Conference 2000.

HOPKIN, J. States, market and corruption: a review of some recent literature. Review of International Political Economy, v. 9, n. 3, p. 574-590, 2002.

ISHAM, J.; KAUFMANN, D.; PRITCHETT, L. Governance and returns on investment: an empirical investigation. Washington: World Bank, 1995. (Policy Research Working Paper, n. 1550).

ISLAM, R. Do more transparent governments govern better? Washington: World Bank, 2003. (Policy Research Working Paper, n. 3077).

KING, R.; LEVINE, R. Financial indicators and growth in a cross section of countries. Washington: World Bank, 1992. (Policy Research Working Paper, 819).

KNACK, S.; KEEFER, P. Institutions and economic performance: cross-country tests using alternative measures. Economics and Politics, v. 7, p. 207-27, 1995.

KRUEGER, A. B.; LINDAHL, M. Education for growth: why and for whom? Journal of Economic Literature, v. 39, n. 4, p. 11011136, Dec. 2001.

LAU, L. J.; JAMISON, D. T.; LOUAT, F. F. Education and productivity in developing countries: an aggregate production function approach. Washington: World Bank, 1991. (Working Paper Series, n. 612). 
LAVERGNE, R.; WOOD, J. Aid effectiveness and non-state partnerships: analytical considerations. Toronto: Canadian International Development Agency, 2006. (CIDA Working Papers).

LEVINE, R.; RENELT, D. A sensitivity analysis of cross-country growth regressions. The American Economic Review, v. 82, n. 4, p. 942-963, 1992.

LINDERT, P. H.; WILLIAMSON, J. G. Does globalization make the world more unequal? In: BORDO, M.; TAYLOR, A. M.; WILLIAMSON, J. G. (Ed.). Globalisation in historical perspective. Chicago: University of Chicago Press, 2003.

LUCAS, R. E. On the mechanics of economic development. Journal of Monetary Economics, v. 22, p. 3-42, July 1988.

MADDISON, A. The world economy: a millennial perspective. Paris: Development Centre Studies, 2001.

MANKIW, N. G. The growth of nations. Brookings Papers in Economic Activity, n. 1, p. 275-326, 1995.

MANKIW, N. G.; ROMER, D.; WEIL, P. A contribution to the empirics of economic growth. Quarterly Journal of Economics, v. 107, n. 2, p. 407-37, 1992.

MEIER, S. G. Leading issues in economic development. Oxford: Oxford University Press, 1995.

MOSLEY, P. Aid, savings and growth revisited. Oxford Bulletin of Economics and Statistics, v. 42, n. 2, p. 79-95, 1980.

MOSLEY, P.; HUDSON, J.; HORRELL, S. Aid, the public sector and the market in less developed countries. Economic Journal, v. 97, n. 387, p. 616-41, 1987.

MURPHY, K.; SHLEIFER, A.; VISHNY, R. Why is rent-seeking so costly to growth? American Economic Review, v.83, n.2, p. 409-414, 1993.

NEHRU, V.; SWANSON, E.; DUBEY, A. New database on human capital stock in developing and industrial countries: sources, methodology and results. Journal of Development Economics, v. 46, p. 379-401, 1995.

NELSON, R. R.; PHELPS, E. Investment in humans, technology diffusion and economic growth. American Economic Review, v. 56, n. 2, p. 69-75, May 1966.

NIE, N. H.; JUNN, J.; STEHLIK-BARRY, K. Education and democratic citizenschip in America. Chicago: Chicago University Press, 1996.

OECD. Human capital investment: an international comparison. Paris: OECD, CENTRE FOR EDUCATIONAL RESEARCH AND INNOVATION. 1998.

PAPANEK, G. F. The effect of aid and other resource transfers on savings and growth in less developed countries. The Economic Journal, v. 82, n. 327, p. 934-950, Sept. 1972.

PRITCHETT, L. Where has all the education gone? The World Bank Economic Review, v. 15, n. 3, p. 367-391, 2001.

PSACHAROPOULOS, G. Returns to education: a further international update and implications. The Journal of Human Resources, v. 20, n. 4, p. 583-604, Aut. 1985. 
PSACHAROPOULOS, G.; PATRINOS, H. A. Returns to investment in education: a further update. Washington: World Bak, 2002. (Policy Research Working Paper, n. 2881).

RADELET, S.; CLEMENS, M.; BHAVNANI, R. Aid and growth: the current debate and some new evidence. Washington DC: Center for Global Development, 2004.

RAJAN, R. G.; SUBRAMANIAN, A. Aid and growth: what does the cross-country evidence really show? Washington DC: International Monetary Fund, 2005. (IMF Working Paper, n. 127).

REINIKKA, R.; SVENSSON, J. Confronting competition: investment response and constraints in Uganda. Washington: World Bank, 1999. (Policy Research Working Paper, n. 2242).

RODRIK, D. One economics, many recipes: globalization, institutions, and economic growth. Princeton: Princeton University Press, 2007.

ROMER, P. M. Human capital and growth: theory and evidence. Conference series on public policy, n. 32, p. 251-286, 1989.

ROODMAN, D. The anarchy of numbers: aid, development, and cross-country empirics. Washington: Center for Global Development (working paper n. 32), 2007.

ROSE-ACKERMAN, S. Corruption and government: causes, consequences, and reform. London: Cambridge University Press, 1999.

SCHUMPETER, J. A. The theory of economic development. Oxford: Oxford University Press, 1961.

SIANESI, B.; VAN REENEN, J. The returns to education: a review of the empirical macro-economic literature. London: Institute of Fiscal Studies, 2002. (Working Paper n. 5).

SPOLAORE, E.; WACZIARG, R. Borders and growth. London: Center for Economic Policy Research, 2005. (CEPR Discussion Papers, n. 5202)

SVENSSON, J. Foreign aid and rent seeking. Journal of International Economics, v. 51, n. 2, p. 327-368, Aug. 2000.

TODARO, M. Economic development. Reading: Addison-Wesley, 2000.

WEISS, T. G. Governance, good governance and global governance: conceptual and actual challenges. Third World Quarterly, v. 21, n. 5, p. 795-814, Oct. 2000.

WILLIAMSON, J. What Washington means by policy reform. In: WILLIAMSON, J (Ed.). Latin American adjustment: how much has happened? Washington: Institute for International Economics, 1990.

WINTERS, L. A. Trade liberalisation and economic performance: an overview. The Economic Journal, v. 114, n. 493, p. F4-F21, 2004.

WORLD BANK. Investing in health. New York: Oxford University Press, 1993 (World Development Report).

WORLD BANK. Development and the next generation. Washington: World Bank, 2007. (World Development Report).

WORLD BANK. Economic growth in the 1990s learning from a decade of reform. Washington: World Bank, 2005. 
WORLD BANK. The challenge of development. Washington: World Bank, 1991. (World Development Report).

WORLD BANK. The State in a changing world. Washington: World Bank, 1997. (World Development Report).

WORLD BANK. Workers in an integrating world. Washington: World Bank, 1995. (World Development Report).

WÖßMANN, L. Specifying human capital. Journal of Economic

Surveys, v. 17, n. 3, p. 239-270, 2003.

\section{Endereço para correspondência}

Gabriella Berloffa

gabriella.berloffa@unitn.it

Giuseppe Folloni

giuseppe.folloni@unitn.it

Ilaria Schnyder

schnyder@sussidiarieta.net 